\title{
Energy dispersion of complex non-isolated vortices
}

\author{
LUO ZheXian ${ }^{1,2}$ \\ ${ }^{1}$ Key Laboratory of Meteorological Disaster of Ministry of Education, Nanjing University of Information Science and Technology, Nanjing \\ 210044, China; \\ ${ }^{2}$ Wuxi Research Center of Environmental Science and Technology, Wuxi 214031, China
}

Received February 24, 2011; accepted June 7, 2011

\begin{abstract}
Energy dispersion is a fundamental scientific problem in the study of natural disasters such as typhoons, heavy rain and earthquakes. The problem has been addressed by both multi-discipline research and forecast studies. The dynamics of isolated circular vortex energy dispersion have been solved. However, the disastrous results of typhoons and heavy rain often occur due to non-isolated circular vortices, the dynamics of which are explored in this paper. The energy dispersion characteristics of non-isolated vortices with complex structural patterns are examined using a linearized nondivergent barotropical vorticity equation model. In the initial field, a tropical cyclone (TC) vortex and a meso-scale vortex coexist, forming a complex structural pattern. An analytic solution based on a Fourier transform and simulations using a two-dimensional model show the following. (1) A wave train of TC-G-D may be created by the energy dispersion where the line connecting the three member centers of the wave train is parallel to the $x$ axis in the case of an initial TC vortex without a meso-scale vortex. (2) A wave train of TC-G-D may also be created by energy dispersion. However, the line connecting the three member centers of the wave train would no longer be parallel to the $x$ axis. Instead, they would form a triangle in the presence of the initial TC vortex with the meso-scale vortex. (3) There is a nonlinear relationship between the initial intensity of the meso-scale vortex and the base angle of the triangle. These results have the potential to be applied in the field of typhoon forecasting.
\end{abstract}

vortex, tropical cyclone (TC), complex structural pattern, energy dispersion, wave train

Citation: $\quad$ Luo Z X. Energy dispersion of complex non-isolated vortices. Chinese Sci Bull, 2011, 56: 2713-2717, doi: 10.1007/s11434-011-4629-2

Multi-disciplinary research into energy dispersion resulting from extreme natural phenomena such as typhoons [1], and heavy rain [2] has long been of interest for weather forecasting and other reasons. For example, Yeh [3] found an analytical solution for one-dimensional perturbation wind velocity $v(x, t)$ and framed energy dispersion theory for atmospheric long waves. This theory explains the lower reach effects of long wavelength perturbations in the westerly belt and has been applied to the practice of weather forecasting for more than fifty years [4].

Tropical cyclones (TC), subtropical vortices and mesoscale vortices in the Mei-yu front are two-dimensional (2D) perturbations [5] that differ from long wavelength perturbations. Chan et al. [6] found an analytical solution for the 2D

email: Luozxma@yeah.net perturbation streamfunction $\psi(x, y, t)$. Luo [7] further found analytically that a synoptic-scale wave train with alternating anti-cyclonic and cyclonic vorticity disturbances, named the TC-G-D wave train, could be created by TC energy dispersion. The analytical result has been confirmed numerically [7]. The characteristics of the TC-G-D wave train can be simulated by both idealized and complex models [8]. The characteristics were also observed in the TCM-90 Field Experiment [9]. Later, the TC-G-D wave train and the relationship between the wave train and TC genesis were found using satellite sounding data [10-13]. This relationship is particularly useful in practical applications.

Previous studies have been constructed using a framework of isolated circular vortex energy dispersion dynamics. In this study, the dynamics of non-isolated circular vortex energy dispersion are explored. Satellite cloud photography 
shows that both isolated and non-isolate circular vortices exist in the atmosphere. A non-isolated circular vortex consists of a circular vortex and one or more meso-scale vortices, forming a complex structural pattern. Such complex non-isolated circular vortices can result in natural disasters, e.g. TC-9914. This complex non-isolated circular vortex TC came ashore at the city of Shamen on 10 October 1999 and caused damages of at least 1900 million Chinese Yuan [14]. Solving the dynamics of such non-isolated circular vortices, including their energy dispersion, is problematic. In this paper we find an analytical solution of the flow evolution using a Fourier transform method, and a numerical solution using 2D simulations. A comparison between the two solutions is made. We then show the characteristics of the complex structure of non-isolated energy dispersion and discuss both the parameter sensibility and application of the techniques.

\section{Model and methods}

The linearized barotropic vorticity equation is

$$
\frac{\partial}{\partial t}\left(\frac{\partial^{2} \psi}{\partial x^{2}}+\frac{\partial^{2} \psi}{\partial y^{2}}\right)+\beta \frac{\partial \psi}{\partial x}=0
$$

where $\psi$ is the geostrophic stream function, and $\beta$ is the meridional gradient of the Coriolis parameter.

An analytical solution is calculated based on a Fourier transform, a method that is used frequently in the Earth sciences $[6,7,15]$.

The Fourier transform of $\psi(x, y, t)$ is

$$
\Psi(K, L, t)=\int_{-\infty}^{\infty} \int_{-\infty}^{\infty} \psi(x, y, t) \mathrm{e}^{-i(K x+L y)} \mathrm{d} x \mathrm{~d} y,
$$

where $K$ and $L$ are the wave numbers in the $x$ and $y$ directions, respectively. Let

$$
\Psi(K, L, t)=\Psi(K, L, 0) \mathrm{e}^{i \omega t},
$$

where $\psi(K, L, 0)$ is the Fourier transform of the initial field $\psi(x, y, 0) . \omega$ is calculated by

$$
\omega=-\frac{\beta K}{K^{2}+L^{2}} .
$$

$\psi(x, y, t)$ can be determined from the following formula:

$$
\psi(x, y, t)=\frac{1}{4 \pi^{2}} \int_{-\infty}^{\infty} \int_{-\infty}^{\infty} \Psi(K, L, 0) \mathrm{e}^{i(K x+L y+\omega t)} \mathrm{d} K \mathrm{~d} L,
$$

where $\psi(K, L, 0)$ is obtained from the initial field $\psi(x, y, 0)$. $\omega$ is determined by (4).

The initial field $\psi(x, y, 0)$ is iteratively determined by the initial relative vorticity field $\xi(x, y, 0)$. Let

$$
\xi(x, y, 0)=\xi_{T}(x, y, 0)+\xi_{S}(x, y, 0),
$$

where $\xi_{T}(x, y, 0)$ corresponds to a TC. Following Chan and Williams [6], $\xi_{T}(x, y, 0)$ is expressed by

$$
\xi_{T}(x, y, 0)=\left(2 V_{\mathrm{m}} / R_{\mathrm{m}}\right) \times\left[1-0.5\left(r / R_{\mathrm{m}}\right)\right] \exp \left[1-\left(r / R_{\mathrm{m}}\right)\right],
$$

where $V_{\mathrm{m}}$ is the maximum wind speed, $R_{\mathrm{m}}$ is the radius of maximum wind, and $r$ is the radial distance from the center of the vortex $\left(x_{0}, y_{0}\right)$, which is at $(0,0) \mathrm{km}$. Set $V_{\mathrm{m}}=20 \mathrm{~m} \mathrm{~s}^{-1}$ and $R_{\mathrm{m}}=100 \mathrm{~km}$.

$\xi_{S}(x, y, 0)$ represents a meso-scale vortex or a meso-scale convectional vortex(MCV). It can be worked out in two steps. First, set

$$
\xi_{A}(x, y, 0)=\xi_{S 0} \sin \left(\frac{x-x_{1}}{x_{2}-x_{1}} \pi\right) \times \sin \left(\frac{y-y_{1}}{y_{2}-y_{1}} \pi\right),
$$

where $\xi_{S 0}$ describes the meso-scale vortex intensity. The mesoscale vortex center $\left(x_{\mathrm{S} 0}, y_{\mathrm{S} 0}\right)=\left(\left(x_{2}+x_{1}\right) / 2.0,\left(y_{2}+y_{1}\right) / 2.0\right) .\left(x_{1}, x_{2}\right)=$ $(-280,0) \mathrm{km},\left(y_{1}, y_{2}\right)=(0,280) \mathrm{km},\left(x_{\mathrm{S} 0}, y_{\mathrm{S} 0}\right)=(-140,140) \mathrm{km}$.

Second, $\xi_{A}(x, y, 0)$ is rotated counterclockwise around the vortex center $\left(x_{\mathrm{S} 0}, y_{\mathrm{S} 0}\right)$ by $45^{\circ}$ to have $\xi_{S}(x, y, 0)$. Substituting $\xi_{T}(x, y, 0)$ and $\xi_{S}(x, y, 0)$ into (6), we get an initial relative vorticity field.

The initial tropical cyclone (TC) vortex defined by $\xi_{T}(x$, $y, 0)$ is an isolated circular vortex (Figure 1(a)). The problem concerning the dispersion characteristics of the energy in
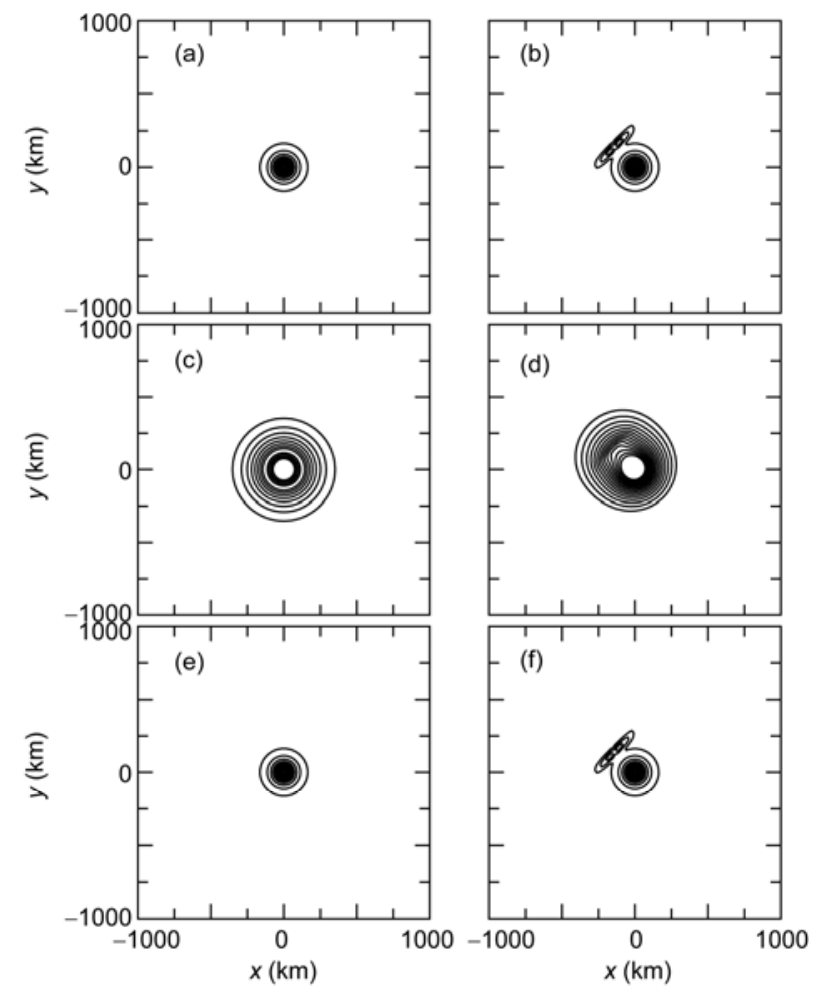

Figure 1 Initial vorticity and streamfunction. (a) $\xi_{T}(x, y, 0)$; (b) $\xi(x, y, 0)$; (c) $\psi_{T}(x, y, 0)$; (d) $\psi(x, y, 0)$; (e) $\xi_{T A}(x, y, 0)$; (f) $\xi_{A}(x, y, 0)$. The contour interval for the vorticity is $2.0 \times 10^{-4} \mathrm{~s}^{-1}$, and for the streamfunction is $2.0 \times$ $10^{4} \mathrm{~m}^{2} \mathrm{~s}^{-1}$. 
such a vertex has been solved. However, the initial vortex defined by $\xi(x, y, 0)$ is a non-isolated vortex (Figure 1(b)).

Note that $\xi_{T}(x, y, 0)=\nabla^{2} \psi_{T}(x, y, 0), \xi(x, y, 0)=\nabla^{2} \psi(x, y$, $0), \psi_{T}(x, y, 0)$, and $\psi(x, y, 0)$ can be determined from $\xi_{T}(x, y$, $0)$ and $\xi(x, y, 0)$ using successive over-relaxation (Figure $1(\mathrm{c}),(\mathrm{d}))$. Then $\xi_{T A}(x, y, 0)$ and $\xi_{A}(x, y, 0)$ can be obtained through the spatial difference method (Figure 1(e), (f). Set $D_{T}=\left(\xi_{T A}(x, y, 0)-\xi_{T}(x, y, 0)\right) / \xi_{T}(x, y, 0), D=\left(\xi_{A}(x, y, 0)-\xi(x\right.$, $y, 0)) / \xi(x, y, 0), D_{T}$ and $D$ can also be obtained. The maximum of both $D_{T}$ and $D$ is less than $0.1 \%$.

For the analytical solution, the model domain is $4000 \times$ $4000 \mathrm{~km}$ with $401 \times 401$ grid points, $\Delta x=\Delta y=10 \mathrm{~km}$. Set $(-200 \leqslant K \leqslant 200,-200 \leqslant L \leqslant 200), \Delta K=\Delta L=1$.

For the numerical solution, the model domain is also $4000 \mathrm{~km} \times 4000 \mathrm{~km}$ with $401 \times 401$ grid points, $\Delta x=\Delta y=$ $10 \mathrm{~km}$. The time step is $180 \mathrm{~s}$, and the model integration is run over $144 \mathrm{~h}$. The central difference Asselin time-filter scheme [16] for time integration is employed at every time step. Periodic boundary conditions are employed on the east-west boundaries and $\partial \psi / \partial t=0$ on the north-south boundaries.

\section{Results and discussion}

Let $\xi_{\mathrm{S}}(x, y, 0)=0$ represent an isolated circular vortex in an initial field. An analytical solution shows that a TC-G-D wave train distinctly appears in the streamfunction field at $t=144 \mathrm{~h}$ and the line connecting the three member centers of the wave train is parallel to the $x$ axis (Figure 2).

Let $\xi_{\mathrm{S}}(x, y, 0)=0$, a numerical solution also clearly shows a TC-G-D wave train that exists in the streamfunction field at $t$ $=144 \mathrm{~h}$, where the line connecting the three member centers

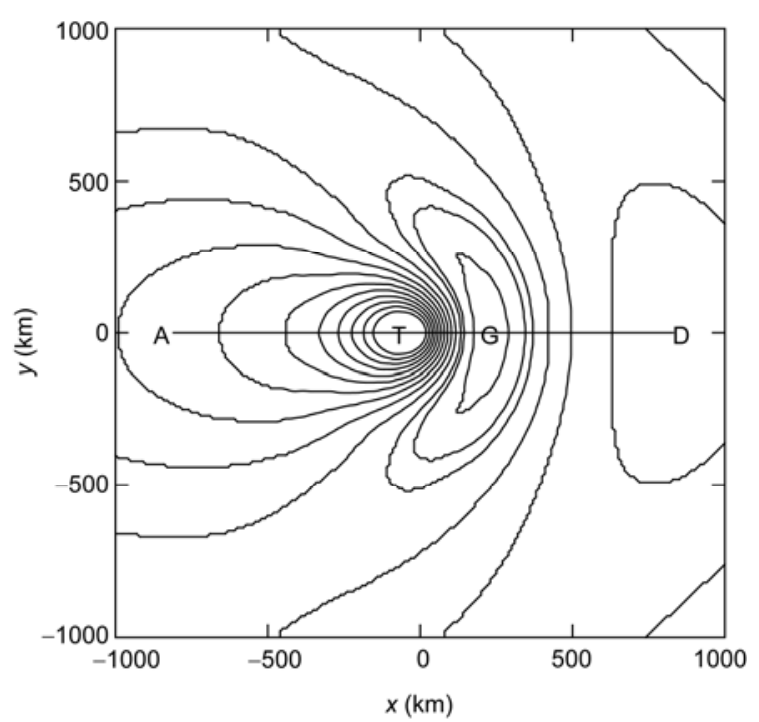

Figure 2 Analytical solution of the streamfunction at $t=144 \mathrm{~h}$ in the case of an isolated circular vortex. The contour interval is $2.0 \times 10^{4} \mathrm{~m}^{2} \mathrm{~s}^{-1}$. of the wave train is also parallel to the $x$ axis (Figure 3). This indicates that the analytical solution is consistent with the numerical solution.

When $\xi_{\mathrm{S}}(x, y, 0)$ is involved, there is a complexly structure non-isolated circular vortex in the initial field. Both analytical and numerical solutions show the TC-G-D wave train that results from TC energy dispersion. However, the line connecting the three member centers of the wave train is no longer parallel to the $x$ axis. Instead, they form a triangle (Figures 4 and 5).

The included angle between the line connecting the TC and $\mathrm{G}$ centers and the $x$ axis is labeled $\alpha$. $\alpha$ equals zero in

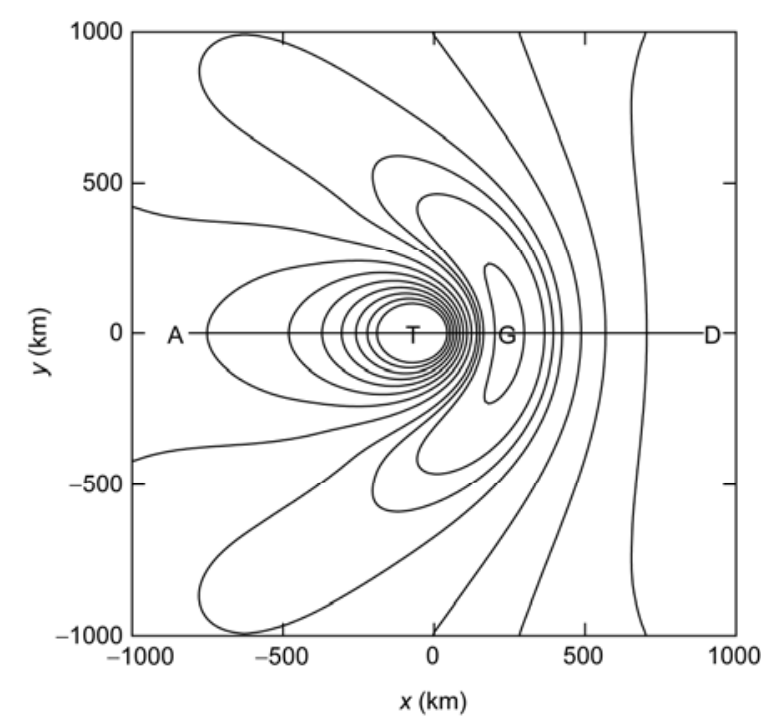

Figure 3 Numerical solution of the streamfunction at $t=144 \mathrm{~h}$ for the case of an isolated circular vortex. The contour interval is $2.0 \times 10^{4} \mathrm{~m}^{2} \mathrm{~s}^{-1}$.

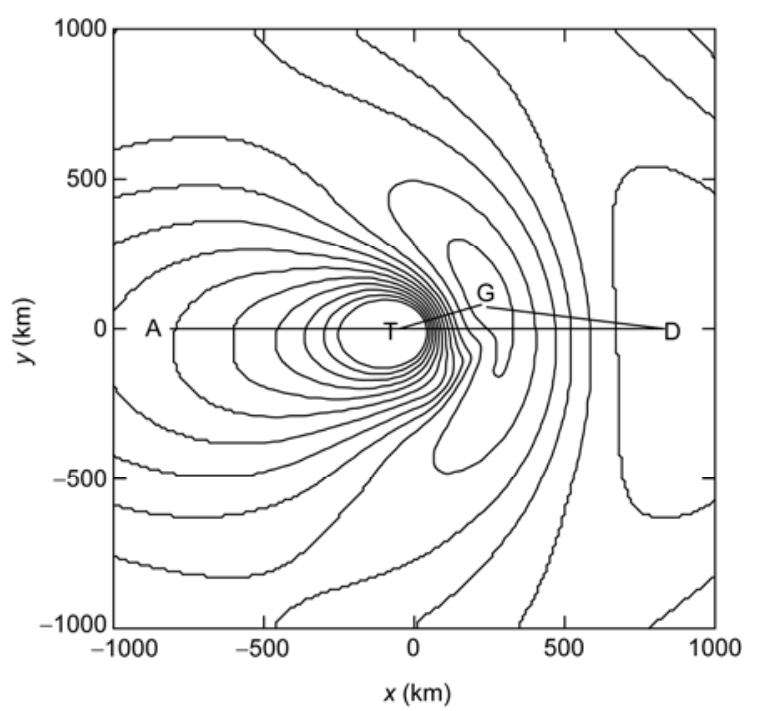

Figure 4 Analytical solution of the streamfunction at $t=144 \mathrm{~h}$ in the case of a complexly structured non-isolated circular vortex. $\xi_{\mathrm{S} 0}=4.0 \times 1.74 \times$ $10^{-4} \mathrm{~s}^{-1}$. The contour interval is $2.0 \times 10^{4} \mathrm{~m}^{2} \mathrm{~s}^{-1}$. 


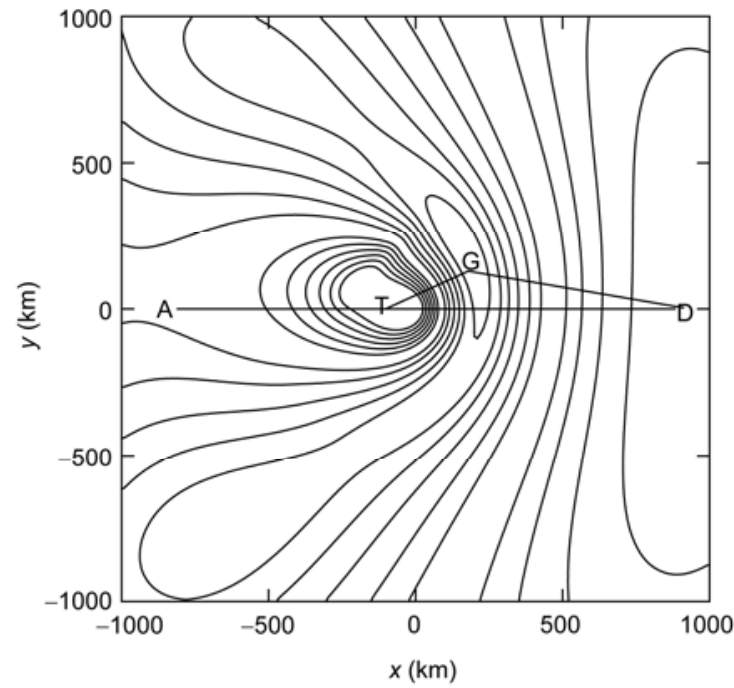

Figure 5 Numerical solution of the streamfunction at $t=144 \mathrm{~h}$ for the case of a complexly structured non-isolated circular vortex. $\xi_{\mathrm{S} 0}=4.0 \times 1.74$ $\times 10^{-4} \mathrm{~s}^{-1}$. The contour interval is $2.0 \times 10^{4} \mathrm{~m}^{2} \mathrm{~s}^{-1}$.

the case of an isolated circular vortex (Figures 2 and 3) whereas $\alpha$ is larger than zero in the case of non-isolated circular vortices (Figures 4 and 5). Thus, the value of $\alpha$ can be used to quantitatively describe the deflection of the connecting line from the $x$ axis. The greater the value of $\alpha$, the larger the deflection, and the greater the difference in the energy dispersion between isolated and non-isolated circular vortices.

$\xi_{\mathrm{S} 0}=4.0 \times 1.74 \times 10 \mathrm{~s}^{-1}$ in Figures 4 and 5 , where $\xi_{\mathrm{S} 0}$ denotes the intensity of the meso-scale vortex involved. Set $\xi_{\mathrm{S} 0}=(1.0,2.0,3.0,5.0,6.0,7.0) \times 1.74 \times 10 \mathrm{~s}^{-1}$ to represent six initial fields. For each of the six initial fields, analytical and numerical solutions and the included angle $\alpha$ are determined for $t=144 \mathrm{~h}$. Results show that $\alpha$ increases with an increase in $\xi_{\mathrm{S} 0}$ when $\xi_{\mathrm{S} 0} \leqslant 4.0 \times 1.74 \times 10 \mathrm{~s}^{-1}$ whereas $\alpha$ is quasi-stationary when $\xi_{\mathrm{S} 0}>4.0 \times 1.74 \times 10 \mathrm{~s}^{-1}$; also the deflection of the connecting line from the $x$ axis always exists when $1.0 \times 1.74 \times 10 \mathrm{~s}^{-1} \leqslant \xi_{\mathrm{S} 0} \leqslant 7.0 \times 1.74 \times 10 \mathrm{~s}^{-1}$ (Figure 6).

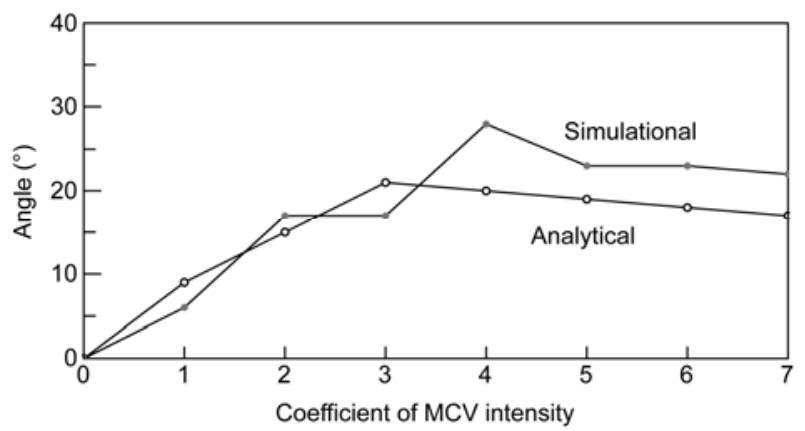

Figure 6 Change of the included angle $\alpha$ between the line connecting the $\mathrm{TC}$ and $\mathrm{G}$ centers and the $x$ axis with $\xi_{\mathrm{s} 0}$. The units for $\xi_{\mathrm{S} 0}$ are $1.74 \times 10^{-4} \mathrm{~s}^{-1}$; the units for $\alpha$ are degrees.

\section{Conclusions}

Atmospheric motion occurs within a Coriolis force field, which is an essential distinction between atmospheric dynamics and general fluid mechanics. The involvement of Coriolis forces results in the atmosphere acting as a dispersion medium that brings about the energy dispersion of largescale weather systems. Yeh [3] proposed the energy dispersion theory of atmospheric long waves as early as the 1940s and advanced weather forecasting theory for the world's westerly belts.

Our understanding of energy dispersion through both TC and subtropical vortices has grown continuously since the 1990s [6-13]. For example, a TC-G-D wave train may be produced by the energy dispersion of an isolated circular vortex, and the line connecting the three member centers of the wave train is parallel to the $x$ axis [7].

Observational studies based on satellite cloud data have discovered that both isolated and non-isolated circular vortices exist in the atmosphere. A non-isolated vortex consists of a single vortex in conjunction with one or more mesoscale vortices, that form a complex structural pattern. The dynamics of non-isolated circular vortex energy dispersion remain to be explored.

An analytical solution based on a Fourier transform and numerical results using a $2 \mathrm{D}$ simulation show that a TC-GD wave train may also be created by energy dispersion associated with a non-isolated circular vortex. However, the line connecting the three member centers of the wave train is no longer parallel to the $x$ axis. Instead, they form a triangle. The G-center is located at the northernmost vertex of the triangle. The results are put in perspective below.

A TC in the western Pacific basin is often located south of a subtropical high. As the TC moves westwards under the steering forces of a subtropical high, it emits Rossby wave energy eastward, forming an anticyclonic system $\mathrm{G}$ east of the TC. Carr and Elsberry [1] found that the anticyclonic system $\mathrm{G}$ may actually connect with the subtropical high in certain conditions. This then results in the extension of the subtropical high southward and an increase in its northsouth extent. The TC track turns from the west to the north under the steering of the changed subtropical high, resulting in the formation of an unusual track.

The evolution of the track can be simulated by a shallow water primitive equation model with an initial field consisting of a TC located south of a subtropical high [17]. However, this track change is on rare occasions. The distance of the anticyclonic system $\mathrm{G}$ from the subtropical high is generally so great that, for the most part, that the connection is not significant.

Our results show that energy dispersion has the following effects in the case of a non-isolated circular vortex (Figure 1(b)). First, the location of an anticyclonic system $G$ is to the north, a favorable position for the connection of the anticyclonic system with the subtropical high. Second, the 
subtropical high extends to the west, which can also make the TC track to the west in some circumstances.

$\mathrm{Li}$ and $\mathrm{Fu}[10]$ clearly distinguished the wave train for a western Pacific TC using satellite sounding data that include scattering surface winds and the state of liquid water. The water content of clouds decreases because of the anticyclonic circulation in the G-area, a region where the probability of TC genesis is low, whereas the water content of clouds increases because of the cyclonic circulation in the $\mathrm{D}$-area, a region where the probability of TC genesis is high.

Results also show that an area where the probability of TC genesis is low will move to the north, but the energy dispersion effect on the cyclonic system D is not obvious when a non-isolated circular vortex is involved.

This study is preliminary. Several problems remain to be solved: (1) the sensitivity of results to the choice of boundary conditions, (2) the sensitivity of results to initial conditions such as the initial distance between the TC and the meso-scale vortex, the meso-scale vortex number, and the meso-scale vortex intensity in a wider value range, (3) the meso-scale vortex intensity effects on the intensity of wave train members TC, G, and D, and (4) the sensitivity of results to uninvolved nonlinear processes. These problems all require further investigation.

We are grateful to three anonymous reviewers for thoughtful comments, which improved the manuscript. This work was supported by the National Natural Science Foundation of China (40775038, 40975036 and 40730948).

1 Carr L E, Elsberry R L. Objective diagnosis of binary tropical cyclone interactions for the western north Pacific basin. Mon Weather Rev, 1998, 126: 1734-1749
2 Cong $\mathrm{C} \mathrm{H}$. On the study of the mechanism of tropical cyclone remote precipitation (in Chinese). Dissertation for the Doctoral Degree. Beijing: The Academy of Meteorological Sciences of China, 2011. 10-17

3 Yeh T C. On energy dispersion in the atmosphere. J Meteorol, 1949, 6: 1-16

4 Wu R S, Dan R Q, Yu Z X, et al. Dyn Meteorol (in Chinese). Shanghai: Shanghai Science and Technology Press, 1983. 75-114

5 Si D, Ding Y H, Liu Y J. Decadal northward shift of the Meiyu belt and the possible cause. Chinese Sci Bull, 2009, 54: 4742-4748

6 Chan J C L, Williams R T. Analytical and numerical studies of the beta-effect in tropical cyclone motion. Part 1: Zero mean flow. J Atmos Sci, 1987, 44: 1257-1265

7 Luo Z X. Energy dispersion effect on TC structure and movement (in Chinese). Acta Meteorol Sin, 1994, 52: 149-156

8 Carr C E III, Elsberry R T. Monsoonal interactions leading to sudden tropical cyclone track changes. Mon Weather Rev, 1995, 123: 265-289

9 Xu X D, Chen L S. Wave train induced by TC energy dispersion in TCM-90 Field Experiment (in Chinese). Acta Meteorol Sin, 1998, 56: 129-138

10 Li T, Fu B. Tropical cyclogenesis associated with Rossby wave energy dispersion of a preexisting typhoon Part I: Satellite data analyses. J Atmos Sci, 2006, 63: 1377-1389

11 Li T, Ge X Y, Wang B, et al. Tropical cyclogenesis associated with Rossby wave energy dispersion of a preexisting typhoon. Part II: Numerical simulations. J Atmos Sci, 2006, 63: 1390-1409

12 Ge X, Li T, Zhou X Q. Tropical cyclone energy dispersion under vertical shears. Geophys Res Lett, 2007, 34: L23807

13 Ge X, Li T, Peng M S. Cyclogenesis simulation of typhoon Prapiroon (2000) associated with Rossby wave energy dispersion. Mon Weather Rev, 2010, 138: 42-54

14 Luo Z H. An investigation into TC-9914 track using satellite data and weather circulations (in Chinese ). Taiwan Straits, 2001, 20: 426-428

$15 \mathrm{Mu} \mathrm{W} \mathrm{F,} \mathrm{Wan} \mathrm{W} \mathrm{X,} \mathrm{Ren} \mathrm{Z} \mathrm{P,} \mathrm{et} \mathrm{al.} \mathrm{Correlation} \mathrm{between} \mathrm{ionospheric}$ longitudinal harmonic components and upper atmospheric tides. Chinese Sci Bull, 2010, 55: 4037-4045

16 Asselin R. Frequency filter for time integrations. Mon Weather Rev, 1972, 109: 18-36

17 Luo Z X. Interaction of dual TC in easterly current south to subtropical high (in Chinese). Acta Meteorol Sin, 2001, 59: 450-458

Open Access This article is distributed under the terms of the Creative Commons Attribution License which permits any use, distribution, and reproduction in any medium, provided the original author(s) and source are credited. 